\title{
Evaporation of Dilute Sodium Dodecyl Sulfate Droplets on a Hydrophobic Substrate
}

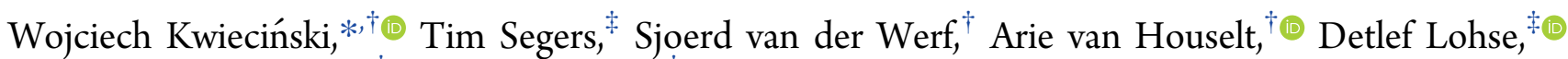 \\ Harold J. W. Zandvliet, ${ }^{\dagger}$ and Stefan Kooij ${ }^{\dagger}$
}

${ }^{\dagger}$ Physics of Interfaces and Nanomaterials group, MESA+ Institute for Nanotechnology and ${ }^{\ddagger}$ Physics of Fluids group, MIRA Institute for Biomedical Technology and Technical Medicine, MESA+ Institute for Nanotechnology, University of Twente, Postbus 217, 7500 AE Enschede, The Netherlands

\section{Supporting Information}

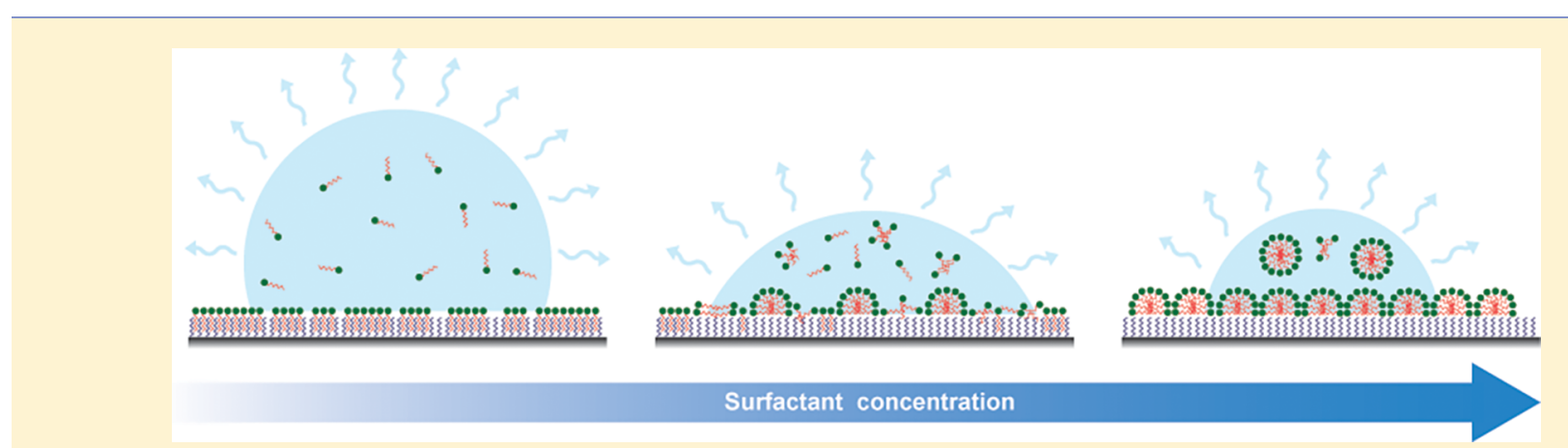

ABSTRACT: Evaporation of surfactant-laden sessile droplets is omnipresent in nature and industrial applications such as inkjet printing. Soluble surfactants start to form micelles in an aqueous solution for surfactant concentrations exceeding the critical micelle concentration (CMC). Here, the evaporation of aqueous sodium dodecyl sulfate (SDS) sessile droplets on hydrophobic surfaces was experimentally investigated for SDS concentrations ranging from 0.025 to $1 \mathrm{CMC}$. In contrast to the constant contact angle of an evaporating sessile water droplet, we observed that, at the same surface, the contact angle of an SDS laden droplet with concentration below $0.5 \mathrm{CMC}$ first decreases, then increases, and finally decreases, resulting in a local contact angle minimum. Surprisingly, the minimum contact angle was found to be substantially lower than the static receding contact angle and decreased with decreasing initial SDS concentration. Furthermore, the bulk SDS concentration at the local contact angle minimum was found to decrease with decrease in the initial SDS concentration. The location of the observed contact angle minimum relative to the normalized evaporation time and its minimum value proved to be independent of both the relative humidity and droplet volume and thus of the total evaporation time. We discuss the observed contact angle dynamics in terms of the formation of a disordered layer of SDS molecules on the substrate at concentrations below 0.5 CMC. The present work underlines the complexity of the evaporation of sessile liquid-surfactant droplets and the influence of surfactant-substrate interactions on the evaporation process.

\section{INTRODUCTION}

Surfactants constitute an enormous group of chemicals that are typically used to reduce the surface tension of liquids. Surfactants usually consist of one or more hydrophobic tails and a polar hydrophilic head. They are commonly used in numerous industries such as food processing, ${ }^{1}$ agriculture, ${ }^{2}$ pharmaceutical, $^{3}$ and inkjet printing. ${ }^{4}$ In many of these applications, surfactant-laden droplets are deposited on a substrate, where they are subsequently left to evaporate., ${ }^{2,4,5}$ There is a vast amount of literature on the evaporation and dissolution of single- and multicomponent sessile droplets. ${ }^{6,7}$ Furthermore, the influence of their surface roughness ${ }^{8-12}$ and wettability ${ }^{13,14}$ on the evaporation process has been extensively investigated in both experimental and theoretical studies. Although the basic physical principles describing droplet evaporation for model cases have been established, many relevant areas remain relatively unexplored, one of which is the influence of surfactants on the evaporation process.

When a soluble surfactant is added to a liquid, its surface tension decreases with increasing surfactant concentration until a threshold value is reached, i.e., the critical micelle concentration (CMC), beyond which the surface tension is not affected any further. ${ }^{15}$ The decrease in the surface tension caused by surfactants typically results in a lower contact angle. The lower contact angle results in a larger droplet perimeter and thus in a longer contact line. The evaporation rate is maximal at the contact line singularity and therefore the addition of surfactants may lead to an increased evaporation rate. ${ }^{16}$ At the same time, insoluble surfactants can significantly

Received: March 20, 2019

Revised: July 3, 2019

Published: July 10, 2019 
reduce the evaporation rate by forming a resistive barrier against liquid molecule diffusion. ${ }^{17}$ Furthermore, a nonuniform surface concentration of surfactants leads to a surface tension gradient, which induces Marangoni flow, in turn resulting in enhanced mixing inside an evaporating droplet. ${ }^{18}$ Surfactantinduced flows can also greatly alter the evaporation process of the droplets loaded with colloidal particles. ${ }^{19}$

Next to surfactant behavior in the liquid bulk, ${ }^{20}$ at the liquid-gas ${ }^{17,18}$ and liquid-liquid ${ }^{21}$ interfaces, the evaporation process is potentially further complicated by surfactant adsorption at the solid substrate, which can lead to dramatic changes in substrate wettability and therewith to phenomena such as superspreading ${ }^{22,23}$ or autophobing. ${ }^{24,25}$ The surfactant adsorption process, the conformation of the adsorbed molecules, and their effect on droplet evaporation are still poorly understood since they proved to be challenging to study. The main difficulty arises from the fact that the adsorbing surfactant layers are usually only a few nanometers in thickness and the adsorption process occurs in a liquid, which limits the available experimental techniques. ${ }^{26-28}$ Furthermore, surfactant adsorption is highly specific for the surfactant-substrate combination and their chemical and physical properties resulting in a huge parameter space. Dynamically changing conditions and a moving contact line, present in the case of an evaporating droplet, complicate the problem even further.

For the extensively studied surfactant sodium dodecyl sulfate (SDS), an anionic surfactant commonly used in industry and research, adsorption at the solid-liquid interface was studied only for static conditions where the whole solid surface was submerged in the surfactant dispersion. ${ }^{27-29}$ It has been shown that the layer of SDS molecules adsorbed to a hydrophobic surface has a maximum thickness for an SDS concentration of approximately $7 \mathrm{mM}$, thus at a concentration below the CMC $(8.2 \mathrm{mM}){ }^{27,29}$ Furthermore, not only the thickness but also the structure of the surfactant layer changes with varying SDS concentration, i.e., from amorphous to micelle-like agglomerates. ${ }^{27}$ Even though the evaporation of SDS-laden droplets has been studied in the past, the influence of surfactant adsorption at the solid-water interface on the evaporation process has not been investigated in full detail. ${ }^{30}$

Here, we studied the evaporation of aqueous SDS-laden sessile droplets from a hydrophobic substrate at SDS concentrations ranging from 0.025 to $1 \mathrm{CMC}$. We aimed to investigate the potential effect of SDS adsorption and layer formation at the solid-liquid interface on the evaporation process.

\section{EXPERIMENTAL DETAILS}

SDS Solution Preparation. Sodium dodecyl sulfate (SDS) with a purity of $\geq 99 \%$ was purchased from Sigma-Aldrich and used without further purification. Water from a Milli- $Q$ system (resistivity $=18.2$ $\mathrm{M} \Omega \mathrm{cm}$ ) was used for solution preparation. Based on literature, the critical micelle concentration (CMC) for an aqueous SDS solution of $8.2 \mathrm{mM}$ was used. ${ }^{31,32}$ The dependence of the surface tension of the SDS solutions on the surfactant concentration has been reported previously. ${ }^{32}$ All solutions were prepared in glass containers that were cleaned first with acetone and then with ethanol and finally rinsed with Milli- $\mathrm{Q}$ water. The solutions were used within $24 \mathrm{~h}$ from their preparation. In the present work, the SDS concentrations are given relative to the CMC. The SDS concentrations mentioned in the description of the evaporation process refer to the initial SDS bulk concentration. The preparation accuracy of the surfactant solutions is estimated to be within 0.002 CMC.
Substrate Preparation. Single-side polished $\mathrm{Si}(100)$ wafers (Okmetic) were diced into $1.5 \times 1.5 \mathrm{~cm}^{2}$ pieces and cleaned in an ultrasonic bath. First in acetone and subsequently in ethanol, both for $10 \mathrm{~min}$. Afterwards, the silicon substrates were dipped in a freshly prepared Piranha solution (3:1 v/v mixture of sulfuric acid (96\%, Merck) and hydrogen peroxide (30\%, Merck)) for approximately 15 min and rinsed thoroughly with Milli- $Q$ water thereafter. Subsequently, the substrates were transferred to a glass vacuum chamber where chemical vapor deposition (CVD) of $1 \mathrm{H}, 1 \mathrm{H}, 2 \mathrm{H}, 2 \mathrm{H}$ perfluorodecyltrichlorosilane (PFDTS, 97\%, Abcr $\mathrm{GmbH}$ ) was performed to hydrophobize the surface. CVD was realized by placing $100 \mu \mathrm{L}$ of PFDTS in a vacuum chamber, which was evacuated and PFDTS was left to evaporate for approximately $12 \mathrm{~h}$. This small amount of PFDTS evaporated within a few minutes. Long reaction times ensured that all the chemical reactions were fully terminated. Later, the substrates were annealed in an oven at $100{ }^{\circ} \mathrm{C}$ for $1 \mathrm{~h}$ to enhance diffusion and the silanization process to ultimately obtain a higher fraction of covalently bound silanes. After cooling down to room temperature, the substrates were submerged in chloroform and put in an ultrasonic bath for 15 min to remove excess PFDTS that was not chemically attached to the surface. As a result of this procedure, we obtained smooth, hydrophobic surfaces with typical root mean square roughness values of only $140 \mathrm{pm}$, as determined by an atomic force microscopy (AFM) (see AFM image in the Supporting Information).

Prior to every experiment, the substrates were cleaned in an ultrasonic bath, first in acetone for $10 \mathrm{~min}$ and subsequently in a $50: 50(\mathrm{v} / \mathrm{v})$ mixture of water and ethanol for another $10 \mathrm{~min}$. After each cleaning step, the substrates were rinsed thoroughly with Milli-Q water and dried under a stream of nitrogen gas.

Measurement Setup. Droplet volumes smaller than $1 \mu \mathrm{L}$ were used to avoid the impact of gravitational force on the shape of the droplets. Depending on the droplet size, two different deposition methods were used. For the $0.5 \mu \mathrm{L}$ droplets, a glass syringe (Hamilton, volume $100 \mu \mathrm{L}$ ) with a cannula needle was used in combination with an automatic dispensing system of a contact angle measurement setup (OCA15+, DataPhysics Instruments GmbH). The same deposition method was used for the receding contact angle measurements. To achieve droplet volumes smaller than $0.5 \mu \mathrm{L}$, a single-nozzle printhead (AD-K-501, Microdrop Technologies GmbH) with a nozzle diameter of $70 \mu \mathrm{m}$ was used. Droplets of approximately $0.5 \mathrm{~nL}$ could be jetted at frequencies up to $100 \mathrm{~Hz}$. By jetting several droplets onto each other, the droplet volume could be controlled over a range smaller than that achievable with the needle deposition method. The droplet volumes studied in this work were varied over 2 orders of magnitude ranging from $3.0 \mathrm{~nL}$ to $0.5 \mu \mathrm{L}$. For both the needle- and printhead-deposited droplets, the evaporation times were approximately 2 orders of magnitude longer than the time required to deposit the droplets. Both the needle and pipette were thoroughly rinsed with Milli-Q water before and after the SDS solutions were introduced.

After droplet deposition, the evaporation process was recorded using a CCD camera (pco.pixelfly, PCO AG) and a lens with adjustable magnification (Navitar). The optical resolution of the images varied between 1 and $4 \mu \mathrm{m} /$ pixel depending on the droplet size. The humidity in the measurement chamber was controlled using a home-made humidity control setup. The setup consisted of a mixing chamber where dry nitrogen gas can enter either directly (to lower the humidity) or through the water column (to increase the humidity). From the mixing chamber, the gas was pumped at a flow rate $(5 \mathrm{~L} / \mathrm{h})$ to the measurement chamber in such a way that it did not flow directly onto the evaporating droplet. The humidity was measured by a sensor placed in the measurement chamber close to the evaporating droplet, and it was used to control the relative humidity (RH) at $44 \pm$ $2 \%$, if not stated otherwise. The temperature in the measurement chamber was fixed at $21 \pm 1{ }^{\circ} \mathrm{C}$.

Image Processing. The recorded images were processed using custom-made Matlab (2017b version, The MathWorks, Inc.) scripts. In the analysis, the droplets were assumed to be axi-symmetric. All droplets were smaller than the capillary length, which allowed us to 
use the spherical cap approximation to determine the contact angle of the droplets by fitting a circle to the droplet's contour that was extracted by the script. The droplet volume was obtained by dividing the droplet into disks exactly one pixel in height and by summing up their volumes. The bulk SDS concentration inside the evaporating droplets was calculated based on the known value of the initial surfactant concentration, the initial droplet volume, and the droplet volume in each time frame extracted from the images. In some cases, the evaporation time $t$ was normalized to the total evaporation time $T$ to aid data comparison, i.e., $\tilde{t}=t / T$. The measurements of the influence of the SDS concentration, humidity, and droplet size on the evaporation process were repeated at least four times, and their contact angle and radius evolutions were averaged.

\section{RESULTS AND DISCUSSION}

Figure 1 shows a comparison between the evaporation process of a pure water droplet and that of a SDS-laden droplet with an
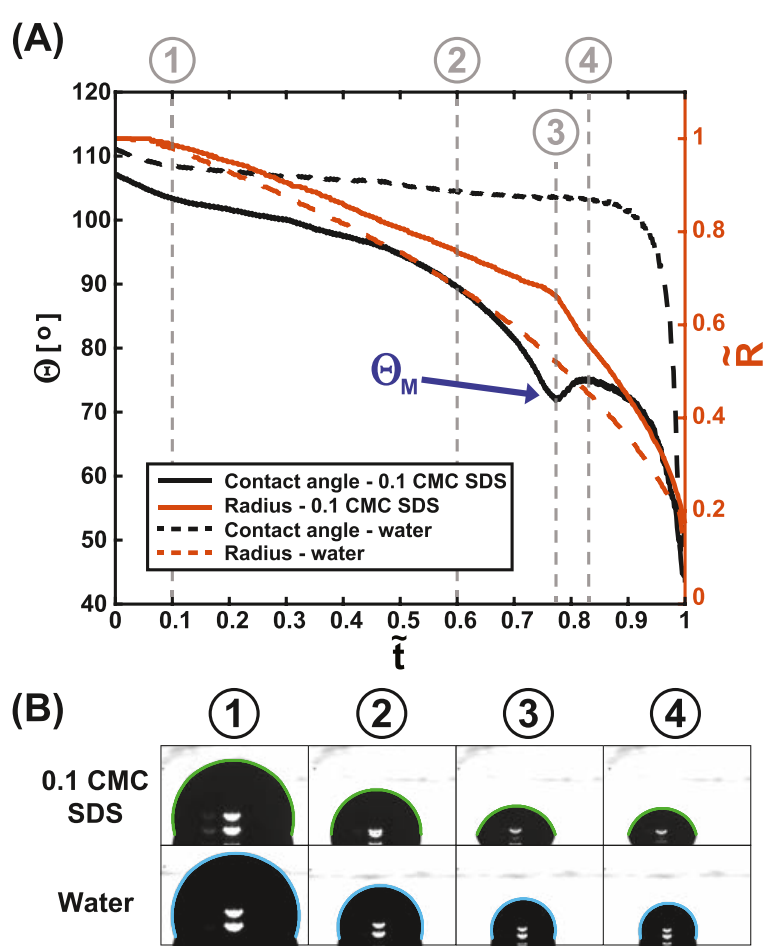

Figure 1. (A) Contact angle (black lines) and normalized radius (red lines) of an evaporating $0.5 \mu \mathrm{L}$ pure water droplet (dashed lines) and of an evaporating $0.5 \mu \mathrm{L}$ SDS laden droplet (0.1 CMC, solid line) as a function of the normalized time. (B) Snapshots of the evaporating SDS-laden droplet (top) and of the pure water droplet (bottom) at different moments in time marked in panel (A). The droplet contours, as found by the image-processing script, are marked by green line for the SDS-laden droplet and in blue for the water droplet. Note that the apparent contour at the bottom edge of the images, which is not fitted, is part of the mirror image, i.e., an optical artefact.

initial bulk concentration of 0.1 CMC. The time is nondimentionalized by the time $T$ it takes to evaporate the whole droplet, $\tilde{t}=t / T$. Note that the water droplet (dashed lines in Figure 1A) evaporates in the constant radius (CR) mode during approximately first $5 \%$ of the total evaporation time. Subsequently, it evaporates in the constant contact angle (CCA) mode throughout $90 \%$ of the total evaporation process, switching to the mixed mode only during the last $5 \%$ of its lifetime. For more information about the various evaporation modes, we refer to refs $33-35$ and the review articles. ${ }^{36,37}$ The SDS-laden droplet (solid lines in Figure 1A) also evaporates in the $\mathrm{CR}$ mode during the first $5 \%$ of the total evaporation time. However, after this initial stage, it switches to a complex mixed mode rather than to the CCA mode as in the case of a pure water droplet. Until approximately half of the total evaporation time, both the contact angle and radius were observed to slowly decrease. Approximately halfway in the evaporation process, the contact angle drops rapidly to reach its minimum value of $\Theta_{M}=72^{\circ}$ (point 3 in Figure 1A,B). Subsequently, the droplet radius starts to decrease rapidly and the contact angle increases to reach a local maximum at $\Theta=75^{\circ}$ (point 4 in Figure 1A). Thus, in contrast to a pure water droplet, the contact angle curve of the SDS-laden droplet has a local contact angle minimum $\Theta_{\mathrm{M}}$.

The dependence of the local contact angle minimum on the SDS concentration is investigated in Figure 2A, i.e., it shows the contact angle evolution of evaporating droplets with initial SDS concentrations $c_{\mathrm{i}}$ ranging from $0.025 \mathrm{CMC}$ up to $1 \mathrm{CMC}$. A local contact angle minimum can be observed for every initial concentration, except for 1 CMC. Furthermore, several interesting trends can be observed with varying initial SDS concentration. First, as expected, the initial contact angle of the droplets decreased with increasing SDS concentration due to a decrease in surface tension. Second, it is observed that the lower the initial SDS concentration, the lower is the contact angle at the local minimum $\Theta_{M}$ (Figure 2B). Especially for $c_{\mathrm{i}}<$ $0.1 \mathrm{CMC}$, the $\Theta$ decreases to values as much as $35^{\circ}$ below those observed during the evaporation of a droplet with $c_{\mathrm{i}}=1$ CMC. Finally, it is observed that the lower the initial SDS concentration, the later the local contact angle minimum occurs during the evaporation process (Figure 2A).

When the contact angle of the droplet is only be governed by its liquid-air interfacial tension, one would expect a minimal contact angle for sessile droplets with $c_{\mathrm{i}} \geq 1 \mathrm{CMC}$, or for droplets whose bulk concentration reaches 1 CMC during evaporation. Thus, the observed local minima cannot be explained from the liquid-air interfacial tension alone. It may be expected that the occurrence of the local contact angle minimum is correlated to the instantaneous SDS concentration $c(t)$ in the evaporating droplet (Figure 2C). Note that the bulk concentration at which $\Theta_{M}$ occurs decreases with decrease in the initial concentration to values as much as $20-50 \%$ below the CMC (Figure 2D). Also note that the local contact angle minimum always occurs at a bulk concentration below 1 CMC. Thus, the local contact angle minimum cannot be explained by micelle formation in the liquid bulk and its onset cannot be correlated to a given SDS bulk concentration.

To investigate whether the occurrence of the local contact angle minimum is dependent only on the bulk surfactant concentration inside the evaporating droplet, the initial droplet volume and the relative humidity were varied. Figure 3A shows the contact angle of the evaporating 0.05 CMC droplets with different initial volumes $(\mathrm{RH}=44 \%)$. The initial droplet volume $V_{\mathrm{i}}$ was varied using the single-nozzle printhead. Surprisingly, despite the fact that the initial volume was varied over 2 orders of magnitude (inset in Figure 3A), no significant differences or trends can be observed. The minor variations in the results can be attributed to small differences in the substrate condition or temperature fluctuations in the room. Furthermore, the total evaporation duration scales linearly with $V_{\mathrm{i}}^{2 / 3}$, which is typical for diffusion-driven evaporation. ${ }^{33}$

The evaporation rate was varied through the relative humidity (Figure 3B). Droplets with $c_{\mathrm{i}}=0.05 \mathrm{CMC}$ and with a constant volume of $50 \mathrm{~nL}$ were used. Note that varying 
(A)

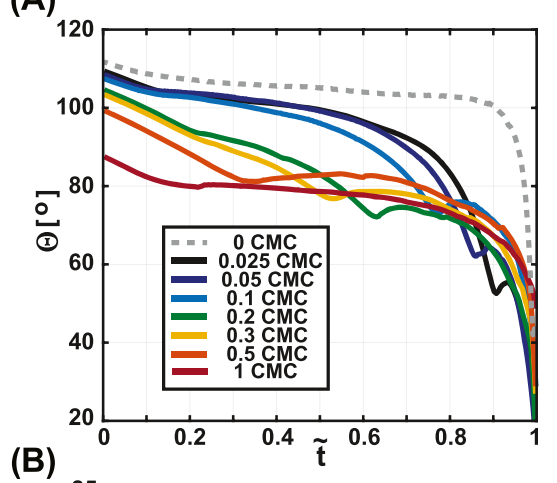

(B)

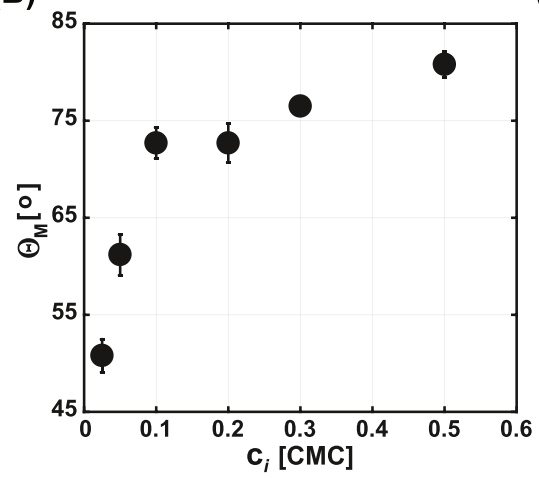

(C)

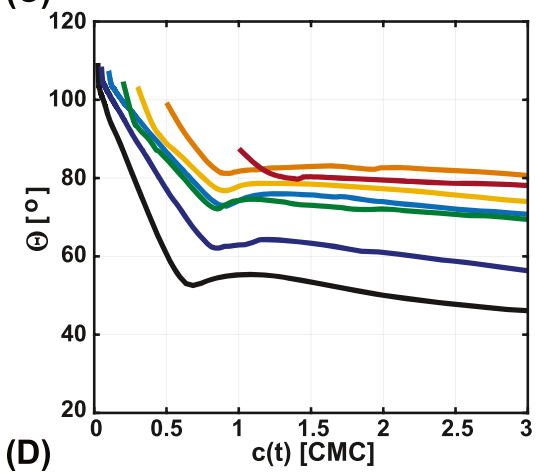

(D)

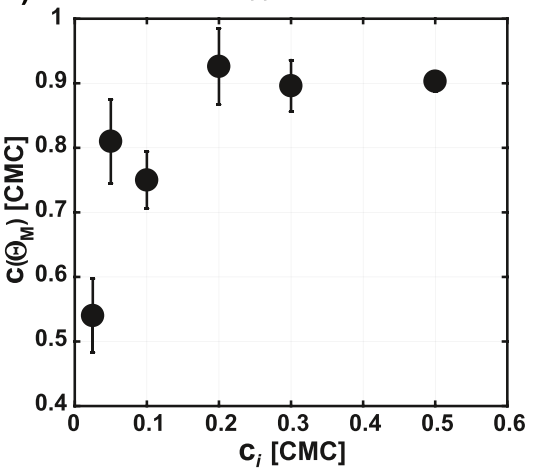

Figure 2. (A) Contact angle averaged over four measurements of evaporating $0.5 \mu \mathrm{L}$ SDS-laden droplets with different initial SDS concentrations as a function of normalized time. (B) Contact angle at the local minimum $\Theta_{M}$ versus the initial SDS concentration. (C) Contact angle averaged over four measurements versus SDS bulk concentration during the evaporation of $0.5 \mu \mathrm{L}$ SDS-laden droplets. (D) Bulk SDS concentration at $\Theta_{\mathrm{T}}$ versus initial SDS concentration. The error bars show standard errors of the mean.

the relative humidity in the measurement chamber did not influence the evaporation process, even though the total evaporation time $T$ varied by a factor of 5 (inset in Figure 3B).

Figure $3 \mathrm{~A}, \mathrm{~B}$ demonstrates the robustness of the local contact angle minimum during the evaporation of dilute SDS-laden droplets, i.e., the phenomenon is independent of the droplet volume and the total evaporation time. This universality suggests that the stochastic contact line pinning due to, e.g., contaminant particles as the mechanism behind the local contact angle minimum can be excluded. Furthermore, it can be concluded that the time scale of the mechanism responsible for the local contact angle minimum is much faster than the time scale of evaporation, since no changes to the droplet evaporation behavior were observed after varying the total evaporation time by almost 2 orders of magnitude.

The change in contact angle with the bulk SDS concentration is now investigated in more detail. Figure 4 shows the derivative of the contact angle $\mathrm{d} \Theta / \mathrm{d} t$ (left axis) and the bulk SDS concentration (right axis) as a function of time for 0.025 and $0.05 \mathrm{CMC}$ droplets. Note that for both cases the contact angle starts to decrease markedly faster when the bulk concentration amounts to approximately $0.15 \mathrm{CMC}$. This strongly suggests that the mechanism responsible for the local contact angle local minimum sets in at bulk SDS concentrations as low as $0.15 \mathrm{CMC}$.

To investigate whether the SDS concentrations around 0.15 $\mathrm{CMC}$ result in the observed contact line pinning, receding contact angle measurements were performed using aqueous SDS solutions with concentrations of $0.05,0.2,0.5$, and 1 $\mathrm{CMC}$. Droplets were deposited on the hydrophobic substrate using the cannula needle and a syringe pump as before. After deposition, the contact line was moved by first increasing the droplet volume to $2 \mu \mathrm{L}$, then decreasing the volume by $0.8 \mu \mathrm{L}$ at a flow rate of $0.1 \mu \mathrm{L} / \mathrm{s}$, and finally increasing the droplet volume again by $0.8 \mu \mathrm{L}$ also at a flow rate of $0.1 \mu \mathrm{L} / \mathrm{s}$. The droplets were imaged during their volume changes to measure the contact angle. The measurement procedure was repeated after 3, 6, and $10 \mathrm{~min}$ to identify time-dependent substrate wettability changes. The humidity inside the measurement chamber was set to $80 \pm 2 \%$, at which point the evaporation was rather small so that the SDS concentration within the droplet can be considered constant.

Figure 5A shows four receding contact angle measurements of the 0.05 CMC solution. The four images overlaid in Figure $5 \mathrm{~B}$ correspond to the moments in time indicated by the numbers in Figure 5A. Note that, independent of the time after droplet deposition, the contact line retracted smoothly. The contact line also retracted smoothly during the initial receding contact angle measurement of the 0.2 CMC solution (Figure 5C). However, the contact angle jumps are clearly observed for the $0.2 \mathrm{CMC}$ measurements repeated at 3, 6, and $10 \mathrm{~min}$; these jumps become more apparent with the passage of time after the first measurement. Also note, a comparison of the droplet-air interface indicated by (3) and (4) in Figure 5D shows that the droplet height increases after sudden increase in contact angle (see Movie in the Supporting Information). The observed contact angle jumps and the corresponding changes in droplet height are a direct proof of contact line pinning. Figure 5E,F shows that the contact lines of the $0.5 \mathrm{CMC}$ and the 1.0 CMC solutions, respectively, again retract smoothly. Thus, Figure 5 demonstrates that, indeed, at the SDS bulk concentrations of approximately $0.2 \mathrm{CMC}$, the wettability of the PFDTS-coated surface is modified, resulting in contact line pinning of the SDS-laden droplet. 

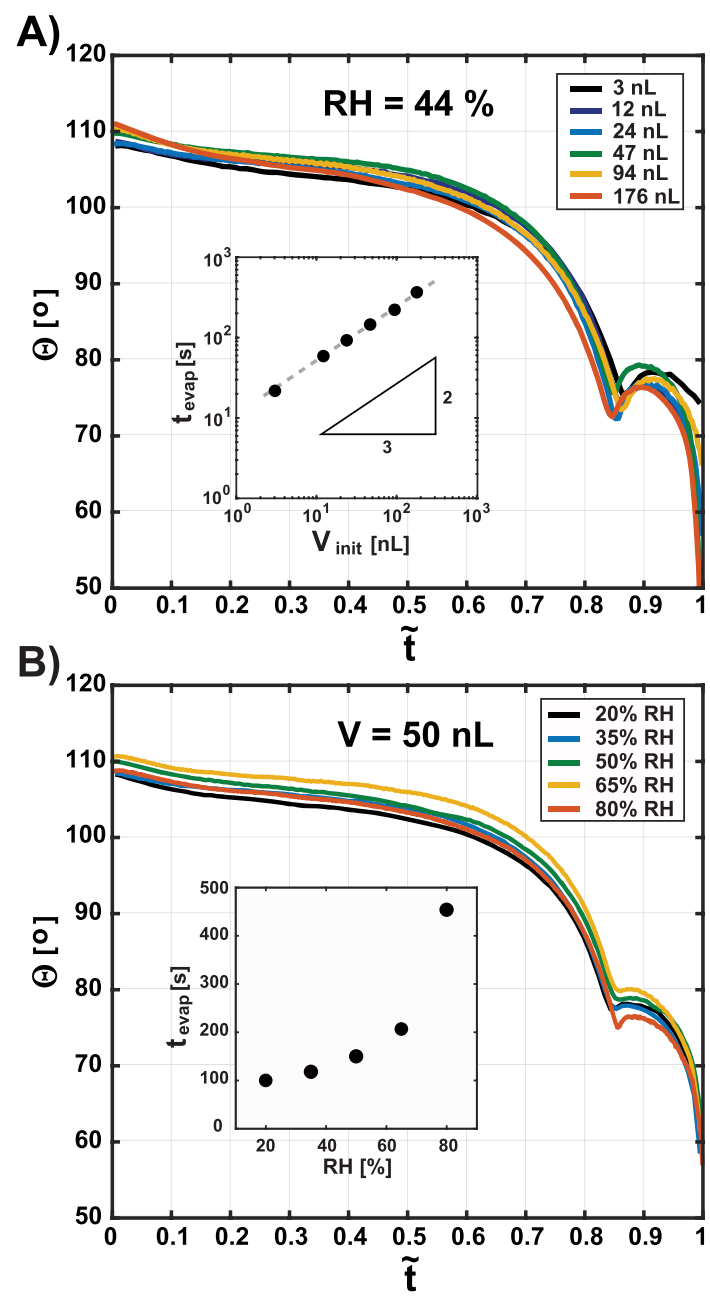

Figure 3. (A) Contact angle averaged over four measurements versus normalized evaporating time for $0.05 \mathrm{CMC}$ droplets of which the initial volume was varied. The relative humidity was $44 \pm 2 \%$. The inset in panel (A) shows the dependence of the total evaporation time $T$ on the initial volume of the droplet $V_{\mathrm{i}}$. Note that $T$ scales as $V_{\mathrm{i}}^{2 / 3}$. (B) Contact angle curves averaged over four measurements as a function of the normalized evaporation time for $50 \mathrm{~nL} 0.05 \mathrm{CMC}$ droplets for varying relative humidity ( $\mathrm{RH})$. The inset in panel (B) shows that the total evaporation time was varied from approximately $100-500 \mathrm{~s}$ through the RH.

SDS Concentration-Dependent Adsorption. The presented experimental results suggest that the local contact angle minimum is induced by a modification of the surface wettability due to adsorbed surfactant molecules. It has been shown that ionic surfactants with long aliphatic carbon chains like $\mathrm{CTAB}$ or SDS adsorb to an amorphous hydrophobic substrate such as PFDTS-coated silicon. ${ }^{26,27}$ Experiments have been performed for static conditions by dipping the substrate in the surfactant solution. The structure of the adsorbed molecules has been shown to strongly depend on the surfactant concentration (Figure 6A). At concentrations below $0.12 \mathrm{CMC}$, the surfactant molecules adsorb on the surface to form an amorphous layer, with the hydrophobic tail pointing toward the molecules coating the surface. At concentrations exceeding $0.12 \mathrm{CMC}$, ordered structures were found to form starting with the sparse formation of domeshaped half-micelles that were found to fully cover the surface at concentrations above $0.73 \mathrm{CMC}$ (Figure 6A). The

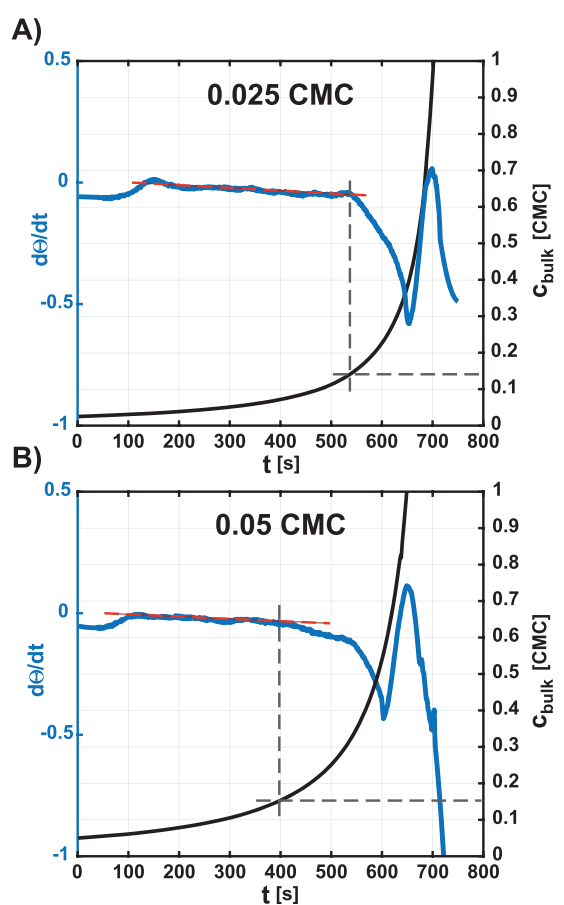

Figure 4. Rate of change of the contact angle with respect to time $\mathrm{d} \Theta / \mathrm{d} t$ (blue lines) and SDS bulk concentration (black lines) as a function of time for evaporating 0.025 CMC (A) and 0.05 CMC (B) droplets. The dashed red lines show the average rate at which $\mathrm{d} \Theta / \mathrm{d} t$ changes during the first part of the evaporation process. The dashed gray lines mark the bulk SDS concentration at which the rate of change of $\mathrm{d} \Theta / \mathrm{d} t$ starts to deviate from that during the first part of the evaporation process.

formation of surface aggregates at concentrations below the $\mathrm{CMC}$, i.e., at concentrations below the threshold for bulk aggregate formation, results from the hydrophobic surface that provides a nucleation site for aggregate formation.

Based on the introduced literature, ${ }^{27}$ a potential mechanism for the observed contact line pinning is discussed. For an evaporating sessile droplet with $c_{\mathrm{i}}<0.1 \mathrm{CMC}$, at first, the SDS molecules form an amorphous layer on the substrate below the droplet. Later, as the surfactant concentration increases through evaporation, the threshold concentration for the surface aggregate formation is reached. However, it is expected that the preadsorbed amorphous surfactant layer hampers the ordered formation of dome-shaped micelles since energy is required to desorb the surfactant monolayer at the location of such a dome-shaped micelle. Therefore, it is speculated that in the case of a dynamically changing surfactant concentration such as that found in an evaporating droplet, disordered surface aggregates are formed (Figure 6B). A disordered layer of adsorbed surfactant aggregates have nonuniform wetting properties through which they can induce contact line pinning. As the evaporation process continues, the surfactant concentration further increases, resulting in a potential reorganization of the disordered surfactant layer into a homogeneous layer with the corresponding homogeneous surface wetting properties. The homogeneous wetting properties will result in a smoothly retracting contact line at a higher contact angle and the absence of contact line pinning, exactly as observed in Figures 1-3 after the appearance of the contact angle minimum. For the sessile droplets with a higher initial surfactant concentrations, i.e., with $0.1<c_{\mathrm{i}}<0.7 \mathrm{CMC}$, the 
(A)

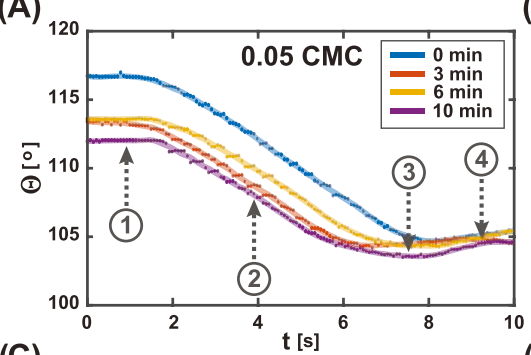

(C)

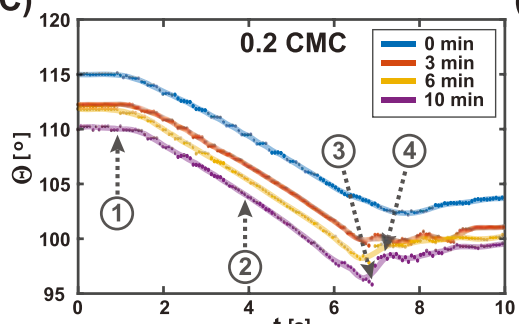

(B)

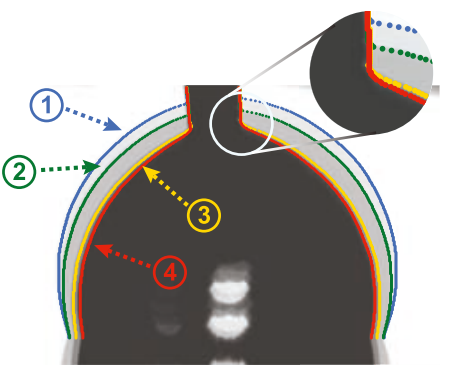

(D)

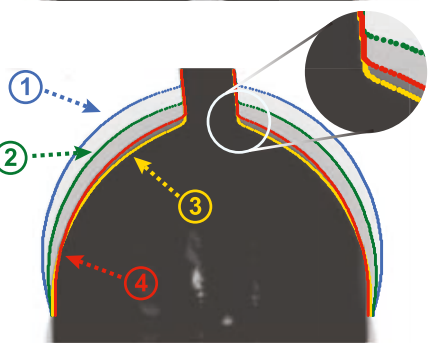

(E)

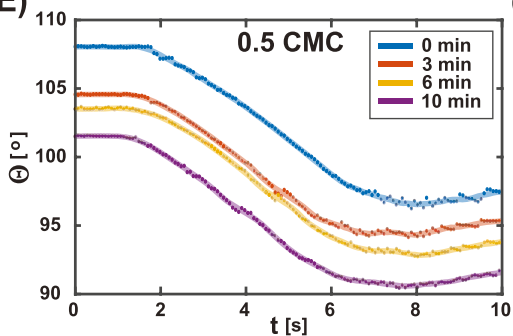

(F)

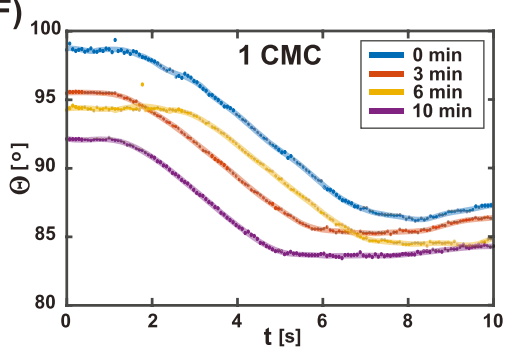

Figure 5. (A, C, E, F) Receding contact angle measurements for the SDS solutions with concentration of $0.05,0.2,0.5$, and 1 CMC. Measurements were performed instantly after the droplet deposition and later after 3, 6, and $10 \mathrm{~min}$ of waiting time. Contact line pinning, growing with time, visible as rapidly increasing contact angle, can be observed for the $0.2 \mathrm{CMC}$ solution. (B, D) Snapshots of the droplet during the measurement (after 10 min of waiting time) for 0.05 and $0.2 \mathrm{CMC}$ solutions in different time points marked on panels (A) and (C). Inset on panel (C) clearly shows the droplet jump caused by the contact line pinning, which is not present on the inset of panel (A).

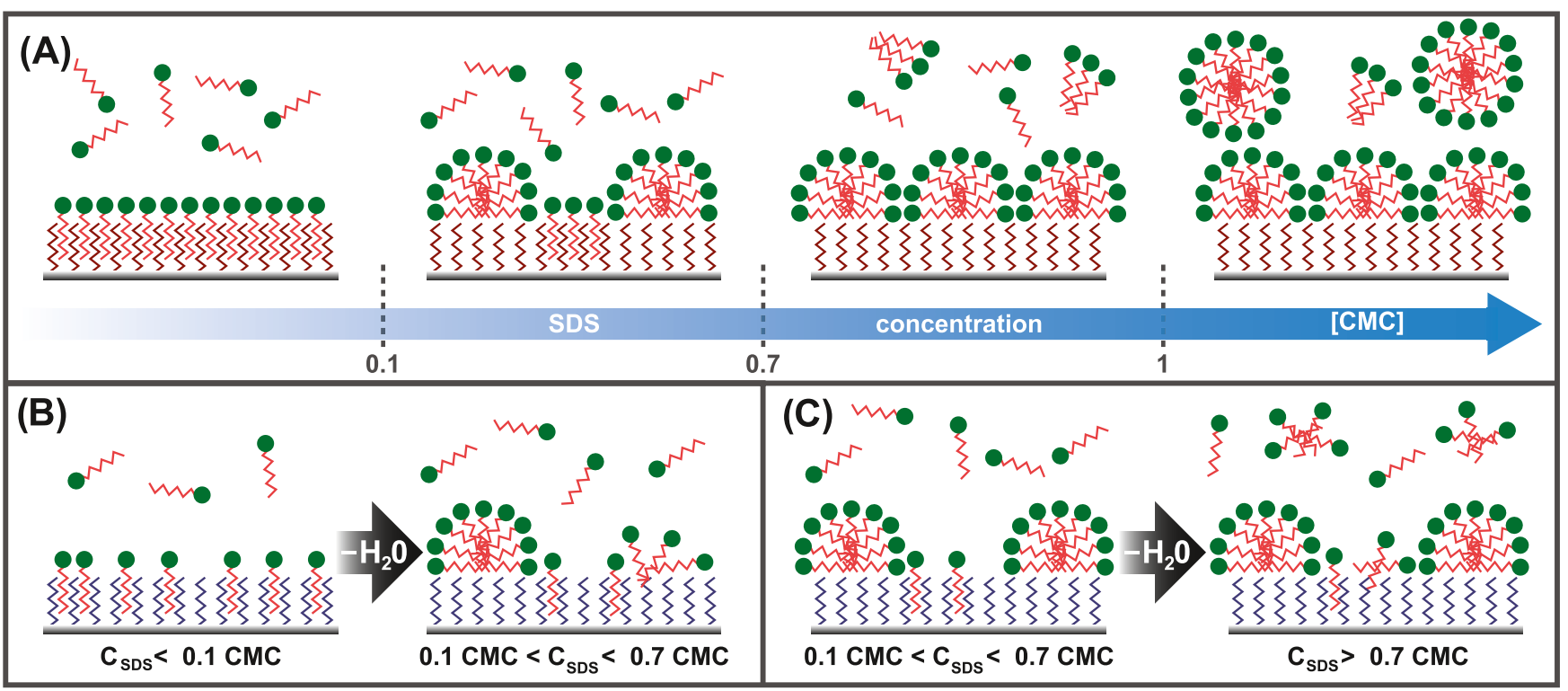

Figure 6. (A) Schematic representation of concentration-dependent SDS adsorption based on the work of Duan et al. ${ }^{27}$ (B) Illustration of disordered SDS aggregate formation during the evaporation of SDS-laden sessile droplets with initial concentrations below 0.1 CMC and (C) that for initial concentrations between 0.1 and 0.7 CMC.

surface is only partly covered with an amorphous layer in the first moments of evaporation (Figure 6C). Therefore, it is expected that $0.1<c_{\mathrm{i}}<0.7 \mathrm{CMC}$ result in a more homogeneous surface wettability. Indeed, this is in perfect agreement with the results shown in Figure 2B, i.e., the contact angle at the local minimum grows with increasing initial concentration of the SDS solution. In an attempt to image the shape of the surfactant structures in the range of low $\left(c_{\mathrm{SDS}}<\right.$ 
0.5 CMC) SDS concentrations, we used AFM imaging in a liquid. Unfortunately, scans appeared blurry, probably because the AFM tip disturbs and interacts with the loosely packed surfactant molecules. A similar effect was reported previously. ${ }^{38,39}$

The receding contact angle measurements showed that the contact line pinning increases with waiting time (Figure 5), which suggests that that disordered adsorption of surfactant molecules is enhanced at a stationary contact line. For the evaporating sessile droplets, no dependence of the local contact angle minimum on the total evaporation time (Figure 3) was observed. This discrepancy is most likely a result of the nonstationary contact line of the evaporating SDS-laden droplets in contrast to the stationary contact line during the receding contact angle measurements.

\section{CONCLUSIONS}

It has been found that the contact angle of an SDS-laden droplet with concentration below 0.5 CMC first decreases, then increases, and finally decreases, resulting in a local contact angle minimum. The minimum contact angle was found to be substantially lower than the static receding contact angle, and it decreased with decreasing initial SDS concentration. Furthermore, the bulk SDS concentration at the local contact angle minimum was found to decrease with a decrease in the initial SDS concentration. The location of the observed contact angle minimum relative to the normalized evaporation time and its minimum value proved to be independent of both the relative humidity and droplet volume. The observed contact angle minimum has been attributed to the contact line pinning induced by the surfactant-concentration-dependent aggregate formation on the substrate below the droplet. The present work highlights the complexity of the evaporation sessile liquid-surfactant droplets and the influence of surfactantsubstrate interactions on the evaporation process.

\section{ASSOCIATED CONTENT}

\section{S Supporting Information}

The Supporting Information is available free of charge on the ACS Publications website at DOI: 10.1021/acs.langmuir.9b00824.

AFM topographical image and the height profile of a PFDTS-coated substrate (PDF)

Movie illustrating the droplet receding measurement (AVI)

\section{AUTHOR INFORMATION}

\section{Corresponding Author}

*E-mail: w.kwiecinski@utwente.nl.

\section{ORCID $\odot$}

Wojciech Kwieciński: 0000-0002-0532-638X

Arie van Houselt: 0000-0002-2254-707X

Detlef Lohse: 0000-0003-4138-2255

\section{Notes}

The authors declare no competing financial interest.

\section{ACKNOWLEDGMENTS}

This work is part of an Industrial Partnership Programme of the Foundation for Fundamental Research on Matter (FOM), which is financially supported by the Netherlands Organisation for Scientific Research (NWO). This research program is co- financed by Océ Technologies B.V., University of Twente, and Eindhoven University of Technology. Additionally, we would like to thank all members of the FIP program for useful discussions and their suggestions. We would also like to thank Guillaume Lajoinie for help with the humidity control setup.

\section{REFERENCES}

(1) Kralova, I.; Sjöblom, J. Surfactants used in food industry: A review. J. Dispersion Sci. Technol. 2009, 30, 1363-1383.

(2) Xu, L.; Zhu, H.; Ozkan, H. E.; Thistle, H. W. Evaporation rate and development of wetted area of water droplets with and without surfactant at different locations on waxy leaf surfaces. Biosyst. Eng. 2010, 106, 58-67.

(3) Singh, Y.; Meher, J. G.; Raval, K.; Khan, F. A.; Chaurasia, M.; Jain, N. K.; Chourasia, M. K. Nanoemulsion: Concepts, development and applications in drug delivery. J. Controlled Release 2017, 252, 2849.

(4) Hanyak, M.; Darhuber, A. A.; Ren, M. Surfactant-induced delay of leveling of inkjet-printed patterns. J. Appl. Phys. 2011, 109, No. 074905.

(5) Pu, N.-W.; Wang, C.-A.; Liu, Y.-M.; Sung, Y.; Wang, D.-S.; Ger, M.-D. Dispersion of graphene in aqueous solutions with different types of surfactants and the production of graphene films by spray or drop coating. J. Taiwan Inst. Chem. Eng. 2012, 43, 140-146.

(6) Tan, H.; Diddens, C.; Lv, P.; Kuerten, J. G. M.; Zhang, X.; Lohse, D. Evaporation-triggered microdroplet nucleation and the four life phases of an evaporating Ouzo drop. Proc. Natl. Acad. Sci. U.S.A. 2016, 8642-8647.

(7) Dietrich, E.; Rump, M.; Lv, P.; Kooij, E. S.; Zandvliet, H. J. W.; Lohse, D. Segregation in dissolving binary-component sessile droplets. J. Fluid Mech. 2017, 812, 349-369.

(8) Chen, X.; Ma, R.; Li, J.; Hao, C.; Guo, W.; Luk, B. L.; Li, S. C.; Yao, S.; Wang, Z. Evaporation of droplets on superhydrophobic surfaces: surface roughness and small droplet size effects. Phys. Rev. Lett. 2012, 109, No. 116101.

(9) Dash, S.; Garimella, S. V. Droplet evaporation dynamics on a superhydrophobic surface with negligible hysteresis. Langmuir 2013, 29, 10785-10795.

(10) Cha, T.-G.; Yi, J. W.; Moon, M.-W.; Lee, K.-R.; Kim, H.-Y. Nanoscale patterning of microtextured surfaces to control superhydrophobic robustness. Langmuir 2010, 26, 8319-8326.

(11) Tsai, P.; Lammertink, R. G. H.; Wessling, M.; Lohse, D. Evaporation-triggered wetting transition for water droplets upon hydrophobic microstructures. Phys. Rev. Lett. 2010, 104, No. 116102.

(12) Susarrey-Arce, A.; Marín, G.; Nair, H.; Lefferts, L.; Gardeniers, J. G. E.; Lohse, D.; van Houselt, A. Absence of an evaporation-driven wetting transition on omniphobic surfaces. Soft Matter 2012, 8, 9765-9770.

(13) Jansen, H. P.; Zandvliet, H. J.; Kooij, E. S. Evaporation of elongated droplets on chemically stripe-patterned surfaces. Int. J. Heat Mass Transfer 2015, 82, 537-544.

(14) Li, Q.; Zhou, P.; Yan, H. J. Pinning-depinning mechanism of the contact line during evaporation on chemically patterned surfaces: a lattice Boltzmann study. Langmuir 2016, 32, 9389-9396.

(15) Adamson, A. W. Physical Chemistry of Surfaces, 3rd ed.; WileyInterscience, 1976; pp 632-633.

(16) Popov, Y. O. Evaporative deposition patterns: Spatial dimensions of the deposit. Phys. Rev. E 2005, 71, No. 036313.

(17) Barnes, G. The effects of monolayers on the evaporation of liquids. Adv. Colloid Interface Sci. 1986, 25, 89-200.

(18) Marin, A.; Liepelt, R.; Rossi, M.; Kähler, C. J. Surfactant-driven flow transitions in evaporating droplets. Soft Matter 2016, 12, 15931600.

(19) Pathak, B.; Hatte, S.; Basu, S. Evaporation dynamics of mixednanocolloidal sessile droplets. Langmuir 2017, 33, 14123-14129.

(20) Nilsson, P. G.; Lindman, B. Mixed micelles of nonionic and ionic surfactants. A nuclear magnetic resonance self-diffusion and proton relaxation study. J. Phys. Chem. A 1984, 88, 5391-5397. 
(21) Gazeau, D.; Bellocq, A. M.; Roux, D.; Zemb, T. Experimental evidence for random surface structures in dilute surfactant solutions. Europhys. Lett. 1989, 9, 447-452.

(22) Rafaï, S.; Sarker, D.; Bergeron, V.; Meunier, J.; Bonn, D. Superspreading: Aqueous surfactant drops spreading on hydrophobic surfaces. Langmuir 2002, 18, 10486-10488.

(23) Stoebe, T.; Lin, Z.; Hill, R. M.; Ward, M. D.; Davis, H. T. Surfactant-enhanced spreading. Langmuir 1996, 12, 337-344.

(24) Bera, B.; Duits, M. H. G.; Cohen Stuart, M. A.; van den Ende, D.; Mugele, F. Surfactant induced autophobing. Soft Matter 2016, 12, 4562-4571.

(25) Afsar-Siddiqui, A. B.; Luckham, P. F.; Matar, O. K. Dewetting behavior of aqueous cationic surfactant solutions on liquid films. Langmuir 2004, 20, 7575-7582.

(26) Manne, S.; Gaub, H. E. Molecular organization of surfactants at solid-liquid interfaces. Science 1995, 270, 1480-1482.

(27) Duan, M.; Wang, H.; Fang, S.; Liang, Y. Real-time monitoring the adsorption of sodium dodecyl sulfate on a hydrophobic surface using dual polarization interferometry. J. Colloid Interface Sci. 2014, 417, 285-292.

(28) Paria, S.; Khilar, K. C. A review on experimental studies of surfactant adsorption at the hydrophilic solid-water interface. Adv. Colloid Interface Sci. 2004, 110, 75-95.

(29) Montgomery, M. E.; Wirth, M. J. Spectroscopic investigation of sodium dodecyl sulfate adsorption on a hydrocarbon monolayer. Langmuir 1994, 10, 861-869.

(30) Doganci, M. D.; Sesli, B. U.; Erbil, H. Y. Diffusion-controlled evaporation of sodium dodecyl sulfate solution drops placed on a hydrophobic substrate. J. Colloid Interface Sci. 2011, 362, 524-531.

(31) Mysels, K. J. Surface tension of solutions of pure sodium dodecyl sulfate. Langmuir 1986, 2, 423-428.

(32) Zhang, J.; Meng, Y. Stick-slip friction of stainless steel in sodium dodecyl sulfate aqueous solution in the boundary lubrication regime. Tribol. Lett. 2014, 56, 543-552.

(33) Picknett, R.; Bexon, R. The evaporation of sessile or pendant drops in still air. J. Colloid Interface Sci. 1977, 61, 336-350.

(34) Stauber, J. M.; Wilson, S. K.; Duffy, B. R.; Sefiane, K. On the lifetimes of evaporating droplets. J. Fluid Mech. 2014, 744, R2.

(35) Stauber, J. M.; Wilson, S. K.; Duffy, B. R.; Sefiane, K. On the lifetimes of evaporating droplets with related initial and receding contact angles. Phys. Fluids 2015, 27, No. 122101.

(36) Cazabat, A.-M.; Guéna, G. Evaporation of macroscopic sessile droplets. Soft Matter 2010, 6, 2591-2612.

(37) Lohse, D.; Zhang, X. Surface nanobubbles and nanodroplets. Rev. Mod. Phys. 2015, 87, 981-1035.

(38) Wanless, E. J.; Ducker, W. A. Organization of sodium dodecyl sulfate at the graphite-solution interface. J. Phys. Chem. A 1996, 100, $3207-3214$

(39) Hong, L.; Nishihara, T.; Hijikata, Y.; Miyauchi, Y.; Itami, K. Unidirectional molecular assembly alignment on graphene enabled by nanomechanical symmetry breaking. Sci. Rep. 2018, 8, No. 2333. 